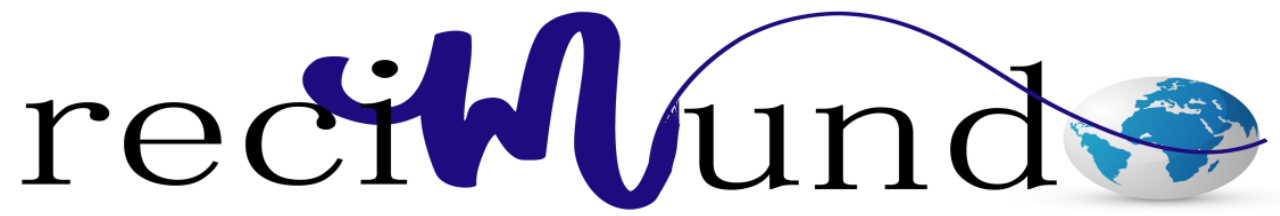

Revista Cientifica Mundo de la Investigación y el Conocimiento

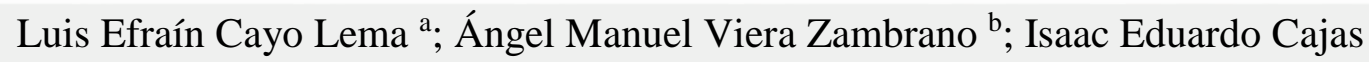
Cayo $^{\text {c; Ruth Susana Hidalgo Guayaquil }}{ }^{\mathrm{d}}$

La docencia y sus competencias en la educación superior

Revista Científica Mundo de la Investigación y el Conocimiento. Vol. 2 núm.2, mayo, ISSN: 2588-073X, 2018, pp. 419-450

DOI: 10.26820/recimundo/2.(2).2018.419-450

Editorial Saberes del Conocimiento

Recibido: 05/12/2017

Aceptado: $15 / 03 / 2018$

a. Universidad Técnica de Cotopaxi; 1uis.cayo@utc.edu.ec

b. Universidad Técnica de Cotopaxi; angel.viera@utc.edu.ec

c. Universidad Técnica de Cotopaxi; isaac.cajas@utc.edu.ec

d. Universidad Técnica de Cotopaxi; ruth.hidalgo@utc.edu.ec 


\section{La docencia y sus competencias en la educación superior}

Vol. 2, núm. 2., (2018)

Luis Efraín Cayo Lema; Ángel Manuel Viera Zambrano; Isaac Eduardo Cajas Cayo; Ruth Susana Hidalgo Guayaquil

\section{RESUMEN}

El estudio del presente trabajo analiza el importancia en la transformación de la educación superior, desde una revisión de literatura con orientación a analizar la docencia y sus competencias, mediante la generación de procesos de enseñanza-aprendizaje orientado a la construcción de "aprender haciendo" en la educación superior; han ayudado a la mejora de una educación de calidad, además resaltar la importancia de las transformaciones de la práctica docente ante las demandas de la sociedad del conocimiento desde finales del siglo pasado, lo cual nos involucra a reconocer que la educación superior se ha transformado y, en consecuencia, el trabajo del docente en el proceso de aprendizaje.

Palabras clave: Transformación de la educación, docencia, competencias, aprender haciendo, sociedad del conocimiento. 


\title{
La docencia y sus competencias en la educación superior
}

Vol. 2, núm. 2., (2018)

Luis Efraín Cayo Lema; Ángel Manuel Viera Zambrano; Isaac Eduardo Cajas Cayo; Ruth

Susana Hidalgo Guayaquil

\begin{abstract}
The study of the present work analyzes the importance in the transformation of higher education, from a review of literature with orientation to analyze teaching and its competences, through the generation of teaching-learning processes oriented to the construction of "learning by doing" in higher education; They have helped to improve quality education, in addition to highlighting the importance of the transformations of the teaching practice faced with the demands of the knowledge society since the end of the last century, which involves us to recognize that higher education has been transformed and, consequently, the work of the teacher in the learning process.
\end{abstract}

Key words: Transformation of education, teaching, competences, learning by doing, knowledge society. 


\section{La docencia y sus competencias en la educación superior}

Vol. 2, núm. 2., (2018)

Luis Efraín Cayo Lema; Ángel Manuel Viera Zambrano; Isaac Eduardo Cajas Cayo; Ruth Susana Hidalgo Guayaquil

\section{Introducción.}

Según, Art. 29.- EI Estado garantizará la libertad de enseñanza, la libertad de cátedra en la educación superior, y el derecho de las personas de aprender en su propia lengua y ámbito cultural. (Constitución de la República del Ecuador, 2008).

La docencia desde la perspectiva teórica del pensamiento dentro de la práctica docente admite que enseñar no se sujeta al aspecto pedagógico, sino que el profesor analiza, reflexiona e interpreta aspectos afectuosos y científicos, propios y de sus alumnos, y en función de ello, realiza su práctica docente; siendo con esto la docencia parte del estudio.

La docencia de calidad en la enseñanza universitaria es un tema de gran importancia en un momento de adaptación para las IES como el actual, donde se esta implementando una reforma universitaria a todos los niveles. Aportando con beneficios que se asocian a una enseñanza universitaria de calidad, siendo una exigencia imprescindible para avalar el adecuado funcionamiento de la educación superior. Sin embargo, consideramos imperioso ahondar en los medios con los que desde el recinto universitario se está afrontando el tema en un proceso de mejora.

Es lógico que la formación académica y social del docente debe ser excelente para poder abordar de manera positiva, una práctica en la que las estrategias que se diseñan para educar se tornan disímiles para cada conjunto de estudiantes y hasta para cada persona en particular. Dichas estrategias deben favorecer también la participación activa de los alumnos en torno a la construcción de sus conocimientos y su propio sentido de vida. Esto, con el propósito de que el conocimiento sea el efecto de un proceso en el cual el docente muestre y motive en sus 


\section{La docencia y sus competencias en la educación superior}

Vol. 2, núm. 2., (2018)

Luis Efraín Cayo Lema; Ángel Manuel Viera Zambrano; Isaac Eduardo Cajas Cayo; Ruth

Susana Hidalgo Guayaquil

educandos, la necesidad de los aprendizajes como herramientas útiles que puedan ayudar a desarrollar potencialidades y competencias que se relacionen con la realidad en que se vive.

La sociedad en su conjunto reclama que el funcionamiento de las universidades esté vinculado a criterios de eficacia, eficiencia y excelencia cada vez mayores (Arranz, 2007).

Hasta la década de 1990 los docentes se centraban básicamente en los procesos de enseñanza. Las reformas de las dos últimas décadas han considerado integrar a la reconfiguración de la naturaleza de la práctica docente, las demandas que le atribuye la sociedad del conocimiento. Es decir, armonizar el proceso de enseñanza y la construcción de protocolos de innovación y creatividad con la ética y el humanismo, todo esto dentro del marco de la globalización.

El expansivo desarrollo de las tecnologías, que las sociedades han experimentado en las últimas décadas, ha marcado los modelos de la educación superior, sus estructuras de gestión y sus impactos. La educación basada en las competencias cobró importancia a inicio del presente siglo, como resultado de la transformación del conocimiento como motor de la economía y factor determinante de la competitividad de los mercados.

Se debe asumir también que los procesos educativos implican un grado de complejidad mayor o menor dependiendo de muchos factores. De modo, que la docencia y sus competencias en la educación superior comprenden todo lo que ha tenido relación con su práctica docente, con su propósito, con la inquietud de mejorarla y con su profesionalización. Por ello, para conocerlas es necesario considerar tres argumentos: el contenido, la clasificación y la formación, es decir, saber qué se enseña, cómo se enseña, a quiénes se enseña y para qué se enseña, desde la 


\section{La docencia y sus competencias en la educación superior}

Vol. 2, núm. 2., (2018)

Luis Efraín Cayo Lema; Ángel Manuel Viera Zambrano; Isaac Eduardo Cajas Cayo; Ruth Susana Hidalgo Guayaquil

perspectiva de las necesidades del desarrollo económico y social y, más específicamente de las demandas del sistema productivo. Cómo es el contexto socio-afectivo de quienes aprenden y enseñan.

Por esta razón, es necesario realizar una investigación que permita determinar la incidencia que ha tenido la docencia y sus competencias en la educación superior en los últimos años.

\section{Metodología.}

El presente artículo científico tiene un enfoque netamente cualitativo, donde se hizo la revisión de literatura acerca de la práctica docente frente a la formación por competencias en la educación superior. Para la realización de la presente investigación, se consideró, información confiable sobre trabajos plasmados, versados en este tema, se utilizaron informaciones bibliográficas, consultas electrónicas, se la obtuvo de libros electrónicos, artículos científicos y revistas.

\begin{tabular}{|c|c|c|}
\hline Libros & Artículos científicos & Revistas \\
\hline 11 & 12 & 10 \\
\hline \multicolumn{3}{|c|}{ 33 bibliografías } \\
\hline
\end{tabular}

En la investigación se utilizaron alrededor de 11 libros donde se encontró información acerca de la educación superior. 12 artículos científicos fueron tomados de referencia. De igual manera los contenidos difundidos en revistas fueron muy importantes, en promedio 33 


\section{La docencia y sus competencias en la educación superior}

Vol. 2, núm. 2., (2018)

Luis Efraín Cayo Lema; Ángel Manuel Viera Zambrano; Isaac Eduardo Cajas Cayo; Ruth

Susana Hidalgo Guayaquil

documentos fueron tomados en consideración acerca de los principales logros en la educación superior representando información importantísima para analizar este tema de manera general.

\section{Desarrollo}

Estudios han demostrado resultados significativos en cuanto a la eficiencia de la educación superior en un país, en el que constantemente se realizan cambios estructurales a la hora de impartirse conocimientos es por ello que.

\section{Importancia de la educación superior en el desarrollo}

UNESCO (2009), Conferencia Mundial sobre la Educación Superior, La propuesta que los participantes en el Debate Temático sobre "La Contribución de la Educación Superior al Sistema Educativo en su Conjunto" podrían formular a la Conferencia para que fuese incluida en su Declaración y Plan de Acción sería la siguiente:

\section{La Conferencia Mundial}

Reconociendo que la educación superior es parte integrante del sistema de educación en su conjunto, en una época en la que la educación se ha convertido en un elemento esencial y perdurable de la vida de las personas y las sociedades en el plano nacional e internacional,

Aceptando que la educación superior tiene una función excepcional en este sistema para contribuir permanentemente a la renovación y ulterior desarrollo de todo él en su conjunto,

Conviene en que, como parte del "nuevo pacto académico" para las universidades del mundo: 


\section{La docencia y sus competencias en la educación superior}

Vol. 2, núm. 2., (2018)

Luis Efraín Cayo Lema; Ángel Manuel Viera Zambrano; Isaac Eduardo Cajas Cayo; Ruth Susana Hidalgo Guayaquil

* las instituciones de educación superior deben adoptar, como parte importante de sus funciones de servicio, el concepto de contribuir mediante asesoramiento, apoyo y cooperación, a la renovación del conjunto del sistema educativo en favor del desarrollo humano sostenible, y más concretamente a la realización de los objetivos de la Educación Básica para Todos proclamados en la Conferencia de Jomtien y confirmados en el Informe Delors;

* se debe conceder una importancia especial a la educación de los docentes como actividad continua, comprendida su formación inicial y su perfeccionamiento profesional a lo largo de toda su carrera, reconociendo la función especial que desempeñan en la renovación de la educación y la necesidad de nuevos modelos de formación y perfeccionamiento;

* teniendo en cuenta la función esencial desempeñada por la educación superior en el descubrimiento, desarrollo y aplicación de conocimientos, las instituciones de educación superior deben desempeñar una función importante en el diálogo permanente del que emana el currículo escolar;

* en el amplio marco de la formación de profesionales, en la que se hallan fundamentalmente implicadas las instituciones de educación superior, se debe conceder una atención y prioridad especiales al perfeccionamiento de los profesionales encargados de la necesaria e importante tarea del aprendizaje a lo largo de toda la vida, con todo lo que ello implica para una cooperación e interpenetración más estrechas entre la educación formal y la no formal;

* las universidades deben prestar una atención especial a los vínculos entre la investigación sobre la educación y la práctica de la educación, teniendo en cuenta la actual 


\section{La docencia y sus competencias en la educación superior}

Vol. 2, núm. 2., (2018)

Luis Efraín Cayo Lema; Ángel Manuel Viera Zambrano; Isaac Eduardo Cajas Cayo; Ruth

Susana Hidalgo Guayaquil

carencia de repercusiones efectivas de la investigación, así como la necesidad extendida de encontrar procesos más eficientes para mejorar la enseñanza;

* reconociendo la gran prioridad que se ha dado en las recientes reuniones de la UNESCO a la educación de mujeres y niñas por la importancia que tiene en el proceso del desarrollo en su conjunto, la educación superior debería tratar de participar en ella dentro de sus propias instituciones y en otras partes;

* la educación superior debe tratar de desempeñar un papel importante en los procesos de análisis y evaluación de los sistemas de educación en proceso de renovación;

* reconociendo los peligros de la intensa competencia provocada por las actuales circunstancias de restricciones financieras, las instituciones de educación superior deben tratar de canalizar la mayor parte de sus energías a través de redes de cooperación que incrementarán el impacto de las instituciones en el plano nacional e internacional.

Manes (1997), la educación superior debe realizar un marketing educativo para desarrollar servicios de enseñanza que respondan a las demandas sociales, para garantizar el equilibrio entre los individuos y las organizaciones.

En la conferencia de la UNESCO (2009), se demuestra que la educación superior ayuda a erradicar la pobreza, fomentar el desarrollo sostenible y alcanzar objetivos acordados en el plano internacional, además en esta misma conferencia se destaca la responsabilidad de la educación superior como comprender y hacer frente a problemas económicos, culturales, sociales y 


\section{La docencia y sus competencias en la educación superior}

Vol. 2, núm. 2., (2018)

Luis Efraín Cayo Lema; Ángel Manuel Viera Zambrano; Isaac Eduardo Cajas Cayo; Ruth Susana Hidalgo Guayaquil

científicos, de promover el pensamiento crítico y la ciudadanía activa, y debe velar por ella los entes interesados, en particular los gobiernos.

En Suiza (2009) en la Conferencia Mundial Sobre Educación Superior, uno de los aspectos primordiales es el de velar por una solución para los problemas económicos, descrito en la Revista Humanun (2012).

\section{La práctica docente en la educación superior}

La práctica docente refleja un esfuerzo de los profesores por mejorar y transitar hacia un aprendizaje más activo de los estudiantes y una responsabilidad importante del profesor para guiarlos. Los profesores mostraron concepciones acerca de la enseñanza y el aprendizaje en las que se refleja que el estudiante juega un papel activo y el profesor sigue como responsable de planear la enseñanza y orientar el aprendizaje. (Cañedo y Figueroa, 2013).

De acuerdo con Fierro, Fortuol y Rosas (2000), La práctica docente, manifiesta a distintas dimensiones, la dimensión personal se refiere a reflexionar sobre el propio quehacer desde la perspectiva que cada uno le imprime como sujeto histórico, capaz de analizar su pasado, resignificar su presente y construir su futuro (pp. 67-68).

La dimensión institucional se considera relevante porque es el espacio en el que el profesor realiza su trabajo cotidiano en el marco de un proyecto educativo (p. 76).

En la dimensión interpersonal, el profesor coadyuva en la creación de un clima para el trabajo basado en el tipo de relaciones que se establecen entre estudiantes y el profesor sobre la base de las diferencias individuales de perspectivas y propósitos (p. 91). 


\section{La docencia y sus competencias en la educación superior}

Vol. 2, núm. 2., (2018)

Luis Efraín Cayo Lema; Ángel Manuel Viera Zambrano; Isaac Eduardo Cajas Cayo; Ruth

Susana Hidalgo Guayaquil

La dimensión social se entiende como el conjunto de decisiones y prácticas de los profesores ante esta diversidad de condiciones culturales y socioeconómicas, que colocan a los alumnos en posiciones distintas frente a la experiencia escolar, y que se convierte en un espacio donde entra en juego de manera más clara la igualdad de oportunidades educativas (p. 107).

La dimensión que refleja los propios valores y la dimensión didáctica, en la cual se centra el estudio, hace referencia al rol del profesor como guía y mediador del contenido y los objetivos del curso a fin de fomentar la construcción de aprendizajes con significado propio a partir de la organización y facilitación del acceso al conocimiento. Reconoce la participación del profesor y el alumno en el proceso educativo, como actores que "hacen posible" las condiciones de enseñanza y aprendizaje. (Fierro, Fortuol y Rosas, 2000)

Los cambios en los paradigmas educativos, propiciados por los avances científicos y tecnológicos en diversos campos, apuntan hacia una restructuración del significado del personal docente y su necesaria constitución como facilitador de procesos de aprendizaje (Duart y Sangrà; 2000; Simonson, Smaldino, Albright \& Zvacek, 2006).

Los cambios paradigmáticos en la formación del profesional de la enseñanza universitaria en el nuevo siglo traen consigo, necesariamente, una concepción diferente de la docencia y de los roles que desempeñan profesores y estudiantes en el proceso de enseñanza-aprendizaje.

De esta manera, la concepción del profesor como transmisor y del estudiante como receptor de conocimientos es sustituida por la concepción del docente como orientador, guía que acompaña al estudiante en el proceso de construcción no sólo de conocimientos, sino también en el desarrollo de habilidades y valores asociados a un desempeño profesional eficiente, ético y 


\section{La docencia y sus competencias en la educación superior}

Vol. 2, núm. 2., (2018)

Luis Efraín Cayo Lema; Ángel Manuel Viera Zambrano; Isaac Eduardo Cajas Cayo; Ruth Susana Hidalgo Guayaquil

responsable, y del estudiante como sujeto de aprendizaje (Díaz y Márquez, 2007; González y González, 2007).

La formación universitaria que parte de modelos basados en una concepción del conocimiento y de los contenidos como los objetivos primordiales del aprendizaje está siendo modificada debido al acelerado cambio de los conocimientos, el acceso y la manera en cómo fluye y, en consecuencia, la provisionalidad de los saberes (Irigoyen, Jiménez, \& Acuña, 2011).

Bok (1992) plantea que cuando los profesores se dedican a la enseñanza suelen inclinarse a favor de la construcción teórica, la generalización y la ideación creativa por encima de la transmisión de habilidades prácticas. Las habilidades son de suma importancia para el ejercicio profesional, enseñar a adquirir esas habilidades puede exigir una inventiva de primer orden, pero rara vez constituye el material sobre el cual se cimientan las reputaciones académicas, pocas veces la transmisión de habilidades puede traducirse en una importante labor académica.

La sociedad actual muestra unos mayores niveles de exigencia en cuanto a la calidad de la formación universitaria y exige a la universidad y a su profesorado que aborden su tarea desde una perspectiva más pedagógica y universitaria y menos burocrática e interesada. Y del mismo modo en que la universidad debe tratar de mostrar rigor en la investigación, también debe buscar la excelencia en la docencia (Martínez y Carrasco, 2006: 22).

(Bain, 2007), define de la siguiente manera al profesor extraordinario: se trata de profesores "que lograron un gran éxito a la hora de ayudar a sus estudiantes a aprender, consiguiendo influir positiva, sustancial y sostenidamente en sus formas de pensar, actuar y sentir". 


\section{La docencia y sus competencias en la educación superior}

Vol. 2, núm. 2., (2018)

Luis Efraín Cayo Lema; Ángel Manuel Viera Zambrano; Isaac Eduardo Cajas Cayo; Ruth

Susana Hidalgo Guayaquil

Mientras otros pueden quedar satisfechos si los estudiantes hacen bien los exámenes, los mejores profesores asumen que el aprendizaje tiene poco sentido si no es capaz de producir una influencia duradera e importante en la manera en que la gente piensa, actúa y siente (Bain, 2007).

"No se trata de adquirir nuevas técnicas de enseñanza, sino de aprovechar la gran base de conocimientos, derivada de la investigación, acerca de la enseñanza y el aprendizaje que tenemos a nuestra disposición" (Biggs, 2006).

Como lo plantea Burbano (2007), citado por Ordóñez, (2009), la sistematización de experiencias "es un proceso teórico y metodológico que a partir del ordenamiento, reflexión crítica, evaluación, análisis e interpretación de la experiencia, pretende conceptuar, construir conocimiento, y a través de su comunicación, orientar otras experiencias para mejorar las prácticas sociales".

La sistematización de experiencias, busca penetrar en el interior de la dinámica de las experiencias, algo así como meterse por dentro de esos procesos sociales vivos y complejos, circulando por entre sus elementos, palpando las relaciones entre ellos, recorriendo sus diferentes etapas, localizando sus contradicciones, tensiones, marchas y contramarchas, llegando así a entender estos procesos desde su propia lógica, extrayendo de allí enseñanzas que puedan aportar al enriquecimiento, tanto de la práctica como de la teoría (Jara Óscar, 2000 en Ordóñez, 2009).

Parra (2003), manifiesta que a partir de las diferentes interpretaciones que da la conducta humana, se han estructurado estrategias y técnicas con el fin de guiar una educación en valores en el aula. 


\section{La docencia y sus competencias en la educación superior}

Vol. 2, núm. 2., (2018)

Luis Efraín Cayo Lema; Ángel Manuel Viera Zambrano; Isaac Eduardo Cajas Cayo; Ruth Susana Hidalgo Guayaquil

Según Camps (1994), “el educador ha de perder el miedo a manifestar sus puntos de vista y sus ideas ante lo que mundialmente ocurre, a criticar lo que juzgue criticable".

Garrido y Doncel (2011, p.7), expresan, "la educación en valores es un ámbito que depende de la competencia de cada maestro o maestra ya que hay maestros que le dan igual de importancia a los valores que a los contenidos de las asignaturas y trabajan ambas cosas y otros que no, que solamente se limitan a dar clases teóricas sobre determinados contenidos sin importarle el desarrollo personal en los alumnos y las alumnas”.

Febres (2013), manifiesta de su investigación que, se sienta un precedente no solo para futuras investigaciones en el campo de la educación vivencial de valores, sino que se conforman unas bases sólidas para proponer estrategias prácticas y sencillas, que el docente pueda ir internalizado en su vida personal y luego verse reflejadas en el campo académico-laboral, que brindarán frutos en la formación humana y por ende, en la estructura integral de los egresados de las universidades.

Para proponer estrategias que sean útiles y aplicadas a corto plazo, es necesario considerar varias ópticas para lograr la efectividad de las mismas, aspectos como la edad del docente, tipo de materia que dicta, nivel cultural, entre otros, que autores como Pérez Esclarín (1997) señalan como variables a tener presente en la formación en valores. Sin embargo la formulación de las siguientes recomendaciones por parte de la autora, se enfocan en un ámbito global de manera de ser adaptadas a cada nivel académico y a cada situación que se presente.

Aunque no todos los aspectos de una sesión de clase son iguales, y obviamente las personas tampoco es importante entender y comprender que el punto de inicio es internalizar y 


\section{La docencia y sus competencias en la educación superior}

Vol. 2, núm. 2., (2018)

Luis Efraín Cayo Lema; Ángel Manuel Viera Zambrano; Isaac Eduardo Cajas Cayo; Ruth

Susana Hidalgo Guayaquil

concientizar la importancia que tiene vivir los valores y principios morales y éticos en los que son modelos de vida en este proceso tan complejo de la educación para que de manera natural, espontánea y auténtica sean vividos y trasmitirlos en cualquier actividad que se realice y en cualquier ámbito de desenvolvimiento de la persona.

López R., (2013), toda profesión es concebida desde un campo disciplinar sea este el de la economía, la medicina, el derecho, la educación; se constituye siempre en busca de un beneficio, ya sea para la manutención individual o familiar, así como para hacer el bien a la sociedad poniendo en juego los saberes y competencias profesionales. El interjuego de estos propósitos que trascienden el utilitarismo individualista es donde la ética toma presencia en el desarrollo de la profesionalidad y, en consecuencia, logra un lugar como actividad socialmente legítima (Cortina, 2000).

López R., (2013), concluye en puntualizar lo siguiente:

a) las finalidades formativas que contiene el discurso universitario a través de su normatividad y proyectos curriculares son resultado de la evolución del pensamiento filosófico y pedagógico, sin embargo, la cultura profesional de sus profesores relativiza su realización, particularmente cuando se observa una debilidad de la ética docente;

b) la dimensión social de la docencia universitaria constituye una identidad ética con el compromiso de hacer de la educación superior un espacio formativo ante los desafíos de $\mathrm{n}$ mundo injusto, la cual se ve disminuida al imponerse una racionalidad técnica-cognitiva en las convicciones de los profesores, $\mathrm{y}$ 


\section{La docencia y sus competencias en la educación superior}

Vol. 2, núm. 2., (2018)

Luis Efraín Cayo Lema; Ángel Manuel Viera Zambrano; Isaac Eduardo Cajas Cayo; Ruth Susana Hidalgo Guayaquil

c) la profesionalidad moral del profesorado se entiende como una visión integral de la docencia universitaria, donde no solo hay que hacer el bien enseñando bien, sino hacer extensivo el principio de beneficencia en todas las dimensiones de la formación, específicamente en aquella que contribuye al desarrollo del ethos de las personas participantes en la relación educativa, cuyas finalidades se ven interferidas cuando la ética de la profesión docente tiene escasa presencia en la configuración cultural de los profesores en la universidad.

Desarrollar el conocimiento acerca de la problemática de los docentes universitarios en la perspectiva de la ética profesional abona a los propósitos de encontrar alternativas en este campo. 


\section{La docencia y sus competencias en la educación superior}

Vol. 2, núm. 2., (2018)

Luis Efraín Cayo Lema; Ángel Manuel Viera Zambrano; Isaac Eduardo Cajas Cayo; Ruth

Susana Hidalgo Guayaquil

\begin{tabular}{|c|c|}
\hline Elemento & Descripción/detalle \\
\hline Contenidos del curso & $\begin{array}{l}\text { Selección y adecuación de contenidos y objetivos } \\
\text { correctamente formulados, organizados y realistas para el } \\
\text { tiempo disponible, precisando los métodos docentes que } \\
\text { permitirán alcanzarlos así como los métodos de evaluación } \\
\text { ajustados a los objetivos. }\end{array}$ \\
\hline $\begin{array}{l}\text { Características de las } \\
\text { presentaciones en } \\
\text { clase }\end{array}$ & $\begin{array}{l}\text { Claridad de las explicaciones, capacidad de comunicación, } \\
\text { entusiasmo, habilidad para plantear y responder preguntas, etc. }\end{array}$ \\
\hline Gestión del curso & $\begin{array}{l}\text { Organización docente, métodos de docencia y evaluación, } \\
\text { accesibilidad para los estudiantes, calidad de la } \\
\text { retroalimentación, coordinación con ayudantes u otros } \\
\text { profesores en su caso, organización de actividades para los } \\
\text { estudiantes tales como proyectos o problemas prácticos, visitas } \\
\text { de campo, elaboración de materiales docentes, etc. }\end{array}$ \\
\hline $\begin{array}{l}\text { Enseñanzas fuera de } \\
\text { clase }\end{array}$ & $\begin{array}{l}\text { Tutorización de estudiantes, planificación y coordinación de } \\
\text { asignaturas y programas de estudio, escribir libros de textos y } \\
\text { otros materiales didácticos, etc. }\end{array}$ \\
\hline $\begin{array}{l}\text { Calidad de los } \\
\text { aprendizajes }\end{array}$ & $\begin{array}{l}\text { Como producto final de una docencia de calidad, incluyendo } \\
\text { adquisición de competencias generales y específicas, } \\
\text { motivación de los estudiantes para seguir estudiando la } \\
\text { materia u otras afines, ajuste de los estudiantes egresados a } \\
\text { perfiles científicos o profesionales actualizados, etc. }\end{array}$ \\
\hline $\begin{array}{l}\text { Actitud profesional y } \\
\text { crítica }\end{array}$ & $\begin{array}{l}\text { Ante la docencia, reflexión y planteamiento explícito y } \\
\text { continuo de la docencia, participación en equipos de trabajo de } \\
\text { finalidad docente, autoevaluación, participación en proyectos } \\
\text { de innovación. }\end{array}$ \\
\hline
\end{tabular}

Cuadro 1. Características de una docencia de calidad, Elaboración a partir de Vizcarro (2003:13), citado por (Castilla, 2011).

\section{Desarrollo de las competencias en la educación superior}

El nuevo perfil del docente en la educación superior, necesita de una formación más exhaustiva en las competencias, abogando por un conocimiento de sus emociones. No solo para ponerla en práctica en su profesión sino también en los diferentes entornos como personales y familiares. Los profesores están permanentemente interaccionando socialmente, con otros 


\section{La docencia y sus competencias en la educación superior}

Vol. 2, núm. 2., (2018)

Luis Efraín Cayo Lema; Ángel Manuel Viera Zambrano; Isaac Eduardo Cajas Cayo; Ruth Susana Hidalgo Guayaquil

compañeros, con sus alumnos, por este motivo se necesita un mayor conocimiento de nuestras emociones para poder conocernos mejor y para saber y prever como piensan y actúan los demás. Abanades, M., (2016).

El espacio de educación superior aboga por que desarrollemos competencias en nuestros alumnos, pero en ocasiones no se ha recibido la formación adecuada para poder realizar esta labor. Algunos actúan de manera ensayo error, otros intentan cultivarse por su cuenta e intentan ponerlo en práctica y otros prefieren actuar como lo hacían hasta el momento, pero la mayoría de los profesores se pronuncian ante la necesidad de tener que conocerla. En los centros de formación y universidades, debería de ser una de las prioridades a la hora de formar a sus docentes. Una formación lo más práctica posible, para que se consolide en el currículo. Abanades, M., (2016).

Informe Delors (1996), el siglo XXI, planteara a la educación que desarrolle a la persona desde una educación integral formada por cuatro pilares. Aplicando estos principios en la educación superior para un nuevo conocimiento:

\section{Aprender a conocer.}

Consiste a la adquisición de conocimientos clasificado y codificado que al dominar los instrumentos del conocimiento y de la comunicación innovando las técnicas de recuperación de la información a través de la aplicación de las tecnologías de la información y de la comunicación. Principalmente se enseñe, aprender a conocer, comprender y descubrir la ciencia. 


\section{La docencia y sus competencias en la educación superior}

Vol. 2, núm. 2., (2018)

Luis Efraín Cayo Lema; Ángel Manuel Viera Zambrano; Isaac Eduardo Cajas Cayo; Ruth

Susana Hidalgo Guayaquil

\section{Aprender hacer}

Los aprendizajes deben evolucionar, no pueden considerarse mera transmisión de prácticas de rutinarias, aunque estos conserven un valor formativo que no debemos desestimar de las diferentes materias, consienta a las personas adultas adquirir competencias personales como trabajar en equipo o grupo, tomar decisiones, relacionarse, crear sinergias y usar de su creatividad.

Aprender a vivir juntos, aprender a vivir con los demás

La idea es que se enseñe a conocernos a nosotros, nuestros sentimientos y emociones, que sea un instrumento para combatir los perjuicios que llevan a enfrentamientos, que nos permitan desarrollar nuestra empatía con los que nos rodean, y con la que también aprendamos a diferenciarnos, a ser críticos, a construir mundos en los que participen ciudadanos con otras opiniones y de todas las culturas.

\section{Aprender a ser}

La educación debe contribuir al desarrollo global de cada persona que nos posibilite desarrollar nuestra libertad, que nos ayude a tener un pensamiento personal y autónomo insertado en una ciudadanía, como la mejor oportunidad de progreso para las sociedades, que sea el espíritu de iniciativa para la creatividad y la innovación. Para disminuir la violencia, el siglo XXI necesitará muy diversos talentos y personalidades, para alcanzar individuos excepcionales nos 


\section{La docencia y sus competencias en la educación superior}

Vol. 2, núm. 2., (2018)

Luis Efraín Cayo Lema; Ángel Manuel Viera Zambrano; Isaac Eduardo Cajas Cayo; Ruth Susana Hidalgo Guayaquil

permita el despliegue completo del hombre; siendo la educación la maduración constante con una experiencia profesional positiva, como medio para alcanzar esa realización.

Huelga decir que los cuatro pilares de la educación descritos no pueden limitarse a una etapa de la vida. Es necesario replantear los tiempos y los ámbitos de la educación, y que se complementen entre si, a fin de que cada persona, durante toda su vida pueda aprovechar al máximo un contexto educativo en constante enriquecimiento.

La filosofía de la formación por competencias tiene dos características centrales: la concepción del sujeto en formación como un sujeto activo que conoce las competencias definidas para el perfil y los criterios de evaluación de las mismas, y que tiene a su disposición las ofertas formativas que le permiten alcanzarlas; y la creación de un ambiente educativo favorable al desarrollo de competencias a través de la utilización de medios didácticos variados. (Mastache, A., 2007).

Cuando la formación no sólo tiene en cuenta los perfiles de competencias previamente identificados sino también la generación de procesos de enseñanza-aprendizaje orientados a la construcción del saber (saber hacer y saber ser) y la movilización de capacidades orientadas a enfrentar nuevas situaciones, se está ante un proceso de formación basado en competencias. Vargas, Casanova y Montanaro, (2001).

Según Tobón (2006), la formación por competencias se asienta en los siguientes principios:

- respuesta a los requerimientos del entorno social y empresarial,

- enfoque hacia la actuación en determinados contextos, 


\section{La docencia y sus competencias en la educación superior}

Vol. 2, núm. 2., (2018)

Luis Efraín Cayo Lema; Ángel Manuel Viera Zambrano; Isaac Eduardo Cajas Cayo; Ruth

Susana Hidalgo Guayaquil

- respuesta a los grandes retos de la humanidad,

- gestión del conocimiento,

- eje en el aprendizaje (no en la enseñanza),

- flexibilidad del diseño curricular,

- autorreflexión como eje del proceso de aprendizaje,

- estrategias para procesar y manejar información como elemento central.

Siguiendo al autor, las principales características del currículo por competencias, que lo diferencian de otros enfoques curriculares, son:

- tiene como base un perfil de competencias,

- el currículo se organiza por módulos y nodos problematizadores,

- los docentes trabajan en equipo,

- está basado en el aprender haciendo, a partir del contexto profesional y la integración de conocimientos, actitudes y procedimientos,

- la evaluación tiene como eje el desempeño.

En el cuadro $\mathrm{N}^{\circ} 1$ se presenta a modo de resumen, las diferentes maneras de expresar los componentes de la competencia docente.

Los cambios educativos en la sociedad del conocimiento exigen que la práctica docente pase de la entrega de información al desarrollo de competencias profesionales para satisfacer las demandas de la sociedad, del sector productivo, y para contribuir de esta manera al desarrollo 


\section{La docencia y sus competencias en la educación superior}

Vol. 2, núm. 2., (2018)

Luis Efraín Cayo Lema; Ángel Manuel Viera Zambrano; Isaac Eduardo Cajas Cayo; Ruth Susana Hidalgo Guayaquil

social y económico del país. Antes de definir estas competencias docentes es fundamental tener en cuenta las características de la sociedad del conocimiento, entre las que destacan:

- El fenómeno de la globalización lleva a afrontar la diversidad.

- Implica nuevas ocupaciones con diferentes formalizaciones del trabajo.

- Presenta dinámicas de comunicación mediadas por el uso de las tecnologías de la información.

- Los nuevos medios de comunicación promueven la formación profesional y nuevas maneras de aprender.

- Demanda destrezas derivadas de la experiencia y una teoría con una amplia perspectiva del contexto social de la educación y del progreso.

En cuanto a los requerimientos de la práctica educativa en las instituciones de educación superior, el papel del docente en el desarrollo de las competencias profesionales (véase Perrenoud en el cuadro 2) tiene múltiples implicaciones de tipo integral y se basa en un modelo sistémico e interdisciplinario.

De tal manera, el docente -en su saber, saber hacer y querer hacer- promueve el desarrollo de competencias profesionales. De ahí, la necesidad de definir las competencias docentes que garanticen la motivación del aprendizaje autónomo y responsable del estudiante mediante el diseño de estrategias que recuperen las condiciones del contexto del ejercicio de la profesión y promuevan la comprensión de la movilización integrada de los saberes relativos a las situaciones complejas asociadas al ejercicio profesional (Roegiers, 2010, p. 172); todo ello en un 


\section{La docencia y sus competencias en la educación superior}

Vol. 2, núm. 2., (2018)

Luis Efraín Cayo Lema; Ángel Manuel Viera Zambrano; Isaac Eduardo Cajas Cayo; Ruth

Susana Hidalgo Guayaquil

ecosistema social caracterizado por la innovación y la creatividad que condiciona la composición de la sociedad y los determinantes del bienestar y el progreso de la humanidad.

\begin{tabular}{|c|c|c|c|c|}
\hline Autor & $\begin{array}{c}\text { Rosa Ma. Esteban } \\
\text { Moreno, Menjivar de } \\
\text { Barbón y Sara Vilma } \\
\text { (2011). }\end{array}$ & $\begin{array}{c}\text { Francisco de Asís } \\
\text { Blas (2007). }\end{array}$ & $\begin{array}{c}\text { Julio H. Pimienta } \\
\text { (2012). }\end{array}$ & $\begin{array}{c}\text { Philippe Perrenoud } \\
\text { (2007). }\end{array}$ \\
\hline País & España & España & México & Francia \\
\hline
\end{tabular}




\section{La docencia y sus competencias en la educación superior}

Vol. 2, núm. 2., (2018)

Luis Efraín Cayo Lema; Ángel Manuel Viera Zambrano; Isaac Eduardo Cajas Cayo; Ruth Susana Hidalgo Guayaquil

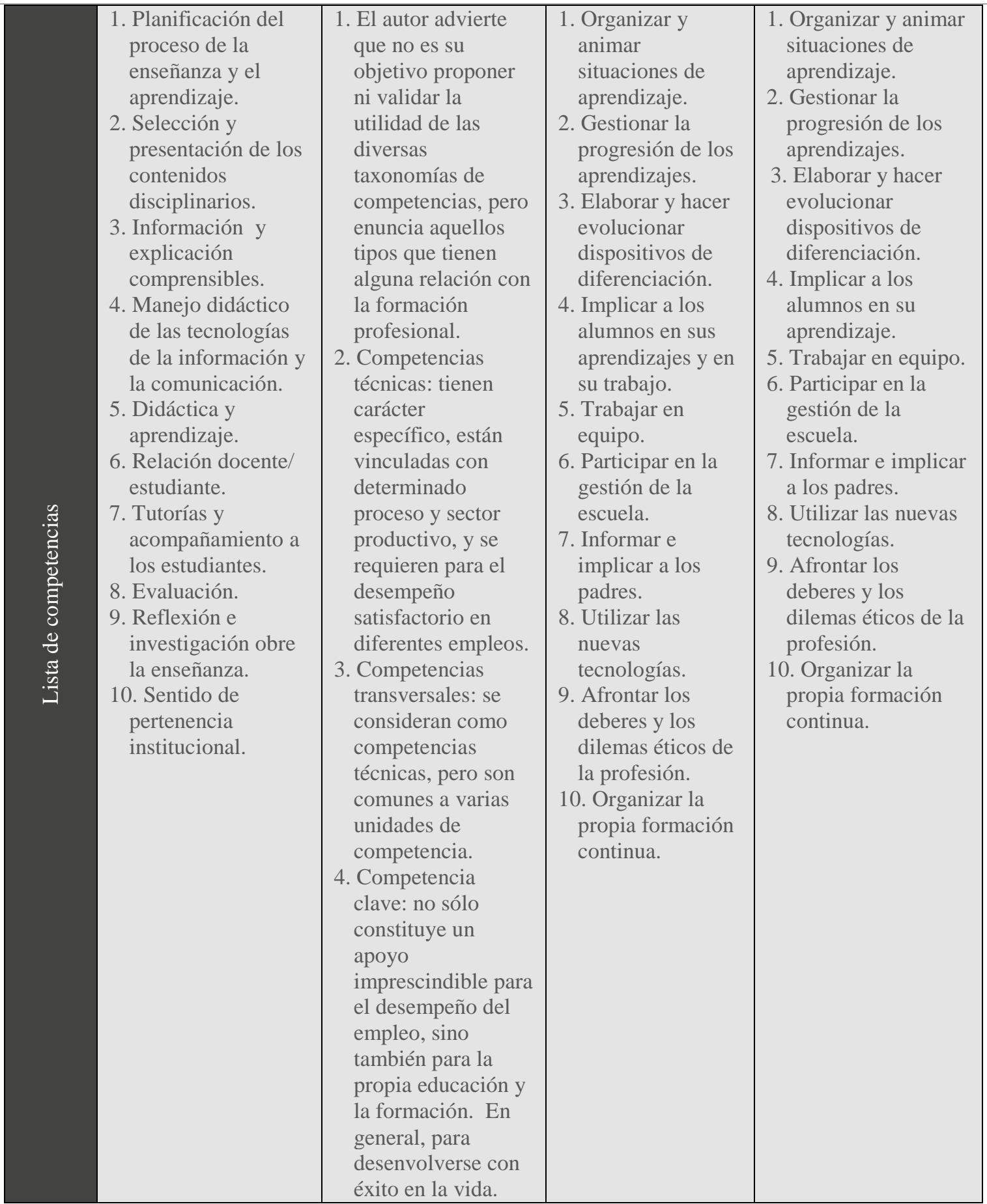

Cuadro 2. Lista de las competencias docentes, citado por Torres R., A.; Badillo G., M.;

Valentín K., N. y Ramírez M., E., (2014). 


\section{La docencia y sus competencias en la educación superior}

Vol. 2, núm. 2., (2018)

Luis Efraín Cayo Lema; Ángel Manuel Viera Zambrano; Isaac Eduardo Cajas Cayo; Ruth

Susana Hidalgo Guayaquil

En cumplimento a la Ley Orgánica de Educación Superior el, Art. 6.- Son derechos de los profesores o profesoras e investigadores o investigadoras de conformidad con la Constitución y esta Ley los siguientes:

a) Ejercer la cátedra y la investigación bajo la más amplia libertad sin ningún tipo de imposición o restricción religiosa, política, partidista o de otra índole:

b) Contar con las condiciones necesarias para el ejercicio de su actividad;

c) Acceder a la carrera de profesor e investigador y a cargos directivos, que garantice estabilidad, promoción, movilidad y retiro, basados en el mérito académico, en la calidad de la enseñanza impartida, en la producción investigativa, en el perfeccionamiento permanente, sin admitir discriminación de género ni de ningún otro tipo;.

d) Participar en el sistema de evaluación institucional:

e) Elegir y ser elegido para las representaciones de profesores/as. e integrar el cogobiemo, en el caso de las universidades y escuelas politécnicas;

f) Ejercer la libertad de asociarse y expresarse;

g) Participar en el proceso de construcción, difusión y aplicación del conocimiento; y,

h) Recibir una capacitación periódica acorde a su formación profesional y la cátedra que imparta, que fomente e incentive la superación personal académica y pedagógica.

En cumplimento a la Ley Orgánica de Educación Superior el, Art. 12.- Principios del Sistema.- El Sistema de Educación Superior se regirá por los principios de autonomía 


\section{La docencia y sus competencias en la educación superior}

Vol. 2, núm. 2., (2018)

Luis Efraín Cayo Lema; Ángel Manuel Viera Zambrano; Isaac Eduardo Cajas Cayo; Ruth Susana Hidalgo Guayaquil

responsable, cogobierno. Igualdad de oportunidades. Calidad, pertinencia, integralidad y autodeterminación para la producción del pensamiento y conocimiento en el marco del diálogo de saberes, pensamiento universal y producción científica tecnológica global. Estos principios rigen de manera integral a las instituciones. Actores, procesos, normas, recursos, y demás componentes del sistema, en los términos que establece esta Ley.

En cumplimento a la Ley Orgánica de Educación Superior el, Art. 13.- Funciones del Sistema de Educación Superior.- Son funciones del Sistema de Educación Superior:

a) Garantizar el derecho a la educación superior mediante la docencia, la investigación y su vinculación con la sociedad, y asegurar crecientes niveles de calidad excelencia académica y pertinencia:

b) Promover la creación, desarrollo, transmisión y difusión de la ciencia, la técnica, la tecnología y la cultura;

c) Formar académicos, científicos y profesionales responsables, éticos y solidarios, comprometidos con la sociedad, debidamente preparados para que sean capaces de generar y aplicar sus conocimientos y métodos científicos, así como la creación y promoción cultural y artística:

d) Fortalecer el ejercicio y desarrollo de la docencia y la investigación científica en todos los niveles y modalidades del sistema:

e) Evaluar, acreditar y categorizar a las instituciones del Sistema de Educación Superior, sus programas y carreras, y garantizar independencia y ética en el proceso. 


\section{La docencia y sus competencias en la educación superior}

Vol. 2, núm. 2., (2018)

Luis Efraín Cayo Lema; Ángel Manuel Viera Zambrano; Isaac Eduardo Cajas Cayo; Ruth

Susana Hidalgo Guayaquil

f) Garantizar el respeto a la autonomía universitaria responsable;

g) Garantizar el cogobierno en las instituciones universitarias y politécnicas;

h) Promover el ingreso del personal docente y administrativo, en base a concursos públicos previstos en la Constitución;

i) Incrementar y diversificar las oportunidades de actualización y perfeccionamiento profesional para los actores del sistema;

j) Garantizar las facilidades y condiciones necesarias para que las personas con discapacidad puedan ejercer el derecho a desarrollar actividad, potencialidades y habilidades:

k) Promover mecanismos asociativos con otras instituciones de educación superior, así como con unidades académicas de otros países, para el estudio análisis, investigación y planteamiento de soluciones de problemas nacionales, regionales, continentales y mundiales:

1) Promover y fortalecer el desarrollo de las lenguas culturas y sabidurías ancestrales de los pueblos y nacionalidades del Ecuador en el marco de la interculturalidad:

m) Promover el respeto de los derechos de la naturaleza, la preservación de un ambiente sano y una educación y cultura ecológica;

n) Garantizar la producción de pensamiento y conocimiento articulado con el pensamiento universal; y.

ñ) Brindar niveles óptimos de calidad en la formación y en la investigación. 


\section{La docencia y sus competencias en la educación superior}

Vol. 2, núm. 2., (2018)

Luis Efraín Cayo Lema; Ángel Manuel Viera Zambrano; Isaac Eduardo Cajas Cayo; Ruth Susana Hidalgo Guayaquil

En cumplimento a la Ley Orgánica de Educación Superior el, Art. 93.- Principio de calidad.- El principio de calidad consiste en la búsqueda constante y sistemática de la excelencia, la pertinencia, producción óptima, transmisión del conocimiento y desarrollo del pensamiento mediante la autocrítica, la crítica externa y el mejoramiento permanente.

En cumplimento a la Ley Orgánica de Educación Superior el, Art. 94.- Evaluación de la calidad.- La Evaluación de la Calidad es el proceso para determinar las condiciones de la institución, carrera o programa académico, mediante la recopilación sistemática de datos cuantitativos y cualitativos que permitan emitir un juicio o diagnóstico, analizando sus componentes, funciones, procesos, a fin de que sus resultados sirvan para reformar y mejorar el programa de estudios, carrera o institución. La Evaluación de la Calidad es un proceso permanente y supone un seguimiento continuo.

CEAACES, (2013), El criterio Academia en esta evaluación alude a las condiciones fundamentales para el ejercicio de una docencia universitaria de calidad. Justamente la denominación de "Academia" tiene por objetivo establecer distinciones con docentes de otros niveles de enseñanza del sistema educativo nacional (básica y bachillerato o sus equivalentes), así como tomar en cuenta la idea de que la docencia universitaria debe constituirse en una verdadera comunidad científica, profesional y artística con autoridad, reconocimiento, legitimidad y debida protección en su medio. Se puede establecer una relación entre la calidad de la enseñanza impartida en las instituciones de educación superior y ciertas características del cuerpo docente, entre las que se destacan:

- Formación académica 


\section{La docencia y sus competencias en la educación superior}

Vol. 2, núm. 2., (2018)

Luis Efraín Cayo Lema; Ángel Manuel Viera Zambrano; Isaac Eduardo Cajas Cayo; Ruth

Susana Hidalgo Guayaquil

\section{- Dedicación docente}

- Carrera docente

- Vinculación con la colectividad

\section{Conclusiones.}

En el presente Artículo se logró establecer, que desde finales del siglo pasado, la educación superior se ha transformado lo cual nos permite reconocer y, en consecuencia, el trabajo del docente en el proceso de aprendizaje.

Por lo tanto el docente se ha visto involucrado a mejorar su desempeño en las actividades de la educación superior, mucho más cuando la educación basada en las competencias cobró importancia a inicio del presente siglo, como resultado de la transformación del conocimiento como motor de la economía y factor determinante de la competitividad de los mercados.

En definitiva el docente está abocado a generar un agradable clima en clases, innovar y provocar la innovación en las prácticas, estableciendo normas así como la responsabilidad en el trabajo, concluyentemente, lo fundamental en el desarrollo de una docencia de excelencia, es que el docente sea accesible y demostrativo, buen observador y competente para organizar un ambiente en que la información perceptible sea comunicada en confianza, comprometido siempre con el cumplimiento y preparación de los contenidos necesarios para ejecutar una determinada asignatura. 


\section{La docencia y sus competencias en la educación superior}

Vol. 2, núm. 2., (2018)

Luis Efraín Cayo Lema; Ángel Manuel Viera Zambrano; Isaac Eduardo Cajas Cayo; Ruth Susana Hidalgo Guayaquil

Del mismo modo, el docente debe entender en la importancia de su cargo para con la institución a la cual se pertenece, consecuentemente preocupado en la formación y cumplimiento de valores, adoptando la mejora continua en su desempeño perfeccionando los procesos y actividades enunciadas en la enseñanza-aprendizaje.

Es tan importante que en el caso de las competencias en la educación superior, el docente debe elegir la mejor práctica, como por ejemplo, innovar en las prácticas de trabajo, puesto que para los estudiantes es más importante la práctica, por lo tanto existe el compromiso en que el docente debe actualizar constantemente los contenidos de las asignaturas que se dictan, es de vital importancia para los docentes que se estimule la realización de clases interactivas con la participación constante de las y los estudiantes, propiciando un agradable ambiente en clases, donde para los docentes como para los estudiantes la práctica docente y el desarrollo de las competencias, se considere primordial en el ejercicio de la docencia, dando lugar a los espacios de consulta y estar dispuesto a atender a los educandos dentro y fuera del aula.

\section{Bibliografía}

Abanades Sánchez, M., (2016). "Nuevo perfil del docente en la educación superior: formación, competencias y emociones”, Universidad Europea de Madrid, España.

Arranz Val, P., (2007), "Los sistemas de garantía de calidad en la Educación Superior en España”. Propuesta de un modelo de acreditación para las titulaciones de Grado en Empresa. Tesis Doctoral, Universidad de Burgos.

Bain, Ken (2007). “Lo que hacen los mejores profesores universitarios”, PUV., Barcelona. Biggs, John (2006). “Calidad del aprendizaje universitario”, Narcea, Madrid. Bok, Derek. (1992). Educación Superior. El Ateneo, Argentina. 


\section{La docencia y sus competencias en la educación superior}

Vol. 2, núm. 2., (2018)

Luis Efraín Cayo Lema; Ángel Manuel Viera Zambrano; Isaac Eduardo Cajas Cayo; Ruth

Susana Hidalgo Guayaquil

CEAACES, (2013), Ecuador: el modelo de evaluación del Mandato 14, Consejo de Evaluación, Acreditación y Aseguramiento de la Calidad de la Educación Superior, 2013. Quito, Ecuador.

Camps, V. (1994). Hacer reforma. Los valores de la educación. Recopilado de: http://www.mercaba.org/Articulos/E/educacion_valores.htm

Cañedo O. Teresa de J., Figueroa R. Alma E., (2013), La práctica docente en educación superior: una mirada hacia su complejidad, www. redalyc.org

Castilla F., (2011), "Calidad docente en el ámbito universitario”, Universidad de Jaén, España, Dialnet

Cortina, A., (2000), "Palabras clave en ética de las profesiones", Editorial Verbo Divino, Navarra.

Delors, J., (1996), "los cuatro pilares de la educación", en la educación encierra un tesoro. Informe a la UNESCO de la comisión internacional sobre la educación para el siglo XXI, Madrid, España.

Díaz, Judith y Márquez, Jeanette. (2007). Estimación del potencial de competencias en la implementación de ejes transversales en instituciones de Educación Superior. Sapiens: Revista Universitaria de Investigación.

Duart, J. y Sangrá, A., (2000), “Aprender en la virtualidad”, https://dialnet.unirioja.es

Febres Natacha, (2013), "Valores en el docente universitario": una exigencia en la actualidad, Revista Educación en Valores, Universidad de Carabobo, Enero - Junio 2013 Vol. 1 N $^{\circ}$ 19

Fierro, Fortuol y Rosas (2000). "Transformando la práctica docente, una propuesta basada en la investigación acción”. Maestros y Enseñanza, Paidós, México.

Garrido L. María A., Doncel B. María J., (2011). “La educación en valores”. Recopilado de: http://www.csi-csif.es/andalucia/modules/mod_ense/revista/pdf/Numero_40

González, Rosa y González, Viviana. (2007). "Diagnóstico de necesidades y estrategias de formación docente en las Universidad". Revista Iberoamericana de Educación. Recuperado de http://www.rieoei.org/1889.htm

Irigoyen, Juan José, Jiménez, Miriam y Acuña, Karla. (2011). "Competencias y Educación Superior". Revista Mexicana de Investigación Educativa (RMIE). 


\section{La docencia y sus competencias en la educación superior}

Vol. 2, núm. 2., (2018)

Luis Efraín Cayo Lema; Ángel Manuel Viera Zambrano; Isaac Eduardo Cajas Cayo; Ruth Susana Hidalgo Guayaquil

López Zavala, R., (2013), “Ética de la docencia universitaria. La dimensión social en la cultura profesional del profesorado”, UAS. México. Dialnet

Manes, J. (1997). Marketing para instituciones educativas, Buenos Aires, Editorial Granica, pp. 17

Martínez, M. y Carrasco, S. (2006), "Propuestas para el cambio docente en la Universidad”, Octaedro, Barcelona. www.octaedro.com

Mastache, Anahí, (2007), “Formar personas competentes”. Desarrollo de competencias tecnológicas y psicosociales, ediciones novedades educativas, Buenos Aires.

Ordóñez P., Zoraida, (2009), “La sistematización en el conocimiento y comprensión de las prácticas sociales”, En: Londoño, Ordóñez, Ried, Enfoques dinámicas y retos en las prácticas sociales con y para jóvenes, Ediciones Unisalle, Bogotá

Parra, M (2003). La educación en valores y su práctica en el aula. Recuperado de: http://www.tendenciaspedagogicas.com/Articulos/2003.pdf

Registro Oficial N 449, (2008), Constitución de la República del Ecuador, Título II, Capítulo II derechos del buen vivir, sección quinta educación, (Art. 29), 20 de octubre.

Registro Oficial Suplemento No 298, (2010), Ley Orgánica de Educación Superior, Título I, Capítulo II, Fines de la Educación Superior, (Art. 6), Capítulo III, Principios del Sistema de Educación Superior, (Art. 12,13), Título V, Calidad de la Educación Superior, Capítulo I, Principio de calidad, (Art. 93, 94), 12 de octubre.

Revista Humanun. (2012). Desarrollo humano mejora pero desigualdades persisten. Recopilado de: http://www.revistahumanum.org/revista

Tobón, F., (2006), “Lineamientos generales para el diseño del currículo por competencias para la educación superior”, Madrid.

Torres R., A.; Badillo G., M.; Valentín K., N. y Ramírez M., E., (2014), "Las competencias docentes": El desafío de la educación superior, innovación educativa, ISSN: 16652673vol.14, número 66.

UNESCO (1998), Conferencia Mundial sobre la Educación Superior, La educación superior en el siglo XXI, visión y acción / la contribución de la educación superior al sistema educativo en su conjunto, Paris, Francia. 


\section{La docencia y sus competencias en la educación superior}

Vol. 2, núm. 2., (2018)

Luis Efraín Cayo Lema; Ángel Manuel Viera Zambrano; Isaac Eduardo Cajas Cayo; Ruth

Susana Hidalgo Guayaquil

UNESCO (2009). Conferencia Mundial sobre la Educación Superior - 2009: La nueva dinámica de la educación superior y la investigación para el cambio social y el desarrollo. Paris, Francia.

Recuperado

de:

http://www.unesco.org/education/WCHE2009/comunicado_es.pdf

Simonson, M., Smaldino, S., Albright, M. \& Zvacek, S., (2006), "Principios de la educacional a distancia y la tecnología instruccional", www.virtualeduca.info

Vargas, F., Casanova, F. y Montanaro, L., (2001), "El enfoque de competencia laboral”: Manual de formación, Montevideo. 